(c) Е.А. Шестакова

ФГБУ Национальный медицинский исследовательский центр эндокринологии Минздрава России, Москва

Сахарный диабет 2 типа (СД2) - заболевание, от осложнений которого во всем мире погибают сотни тысяч человек в год. С каждым годом появляются новые препараты, а почти каждое десятилетие - классы препаратов, направленные на снижение гликемии. К сожалению, несмотря на это, многие пациенты с СД2 не достигают компенсации углеводного обмена. Согласно большинству рекомендаций по лечению СД2, первой линией терапии остается метформин. Выбор того или иного препарата для формирования комбинации с метформином осуществляется в соответствии с международными и локальными руководствами, часто оставляющими выбор второй линии терапии за врачом. Поэтому актуальным остается вопрос определения целевых групп пациентов сахароснижающего препарата второй линии. В данном обзоре представлены данные о влиянии различных групп сахароснижающих препаратов на прогрессирование микро- и макрососудистых осложнений СД. Рассматривается целесообразность ранней интенсификации сахароснижающей терапии с целью активации «метаболической памяти» - механизма замедления прогрессирования осложнений СД. Длительное поддержание компенсации углеводного обмена в дебюте заболевания с помощью комбинации сахароснижающих препаратов может служить основой первичной профилактики сердечно-сосудистых исходов у пациентов с СД.

кЛЮчЕВЫЕ СлОВА: сахарный диабет 2 типа; сахароснижающая терапия; иДПП-4; иНГЛТ-2; микрососудистые осложнения; макрососудистые осложнения; метаболическая память

\title{
SECOND LINE THERAPY IN TYPE 2 DIABETES: LEGACY EFFECT ACTIVATION
}

\author{
(c) Ekaterina A. Shestakova
}

Endocrinology research centre, Moscow, Russia

Type 2 diabetes causes hundred thousand deaths worldwide every year. Though new antidiabetic drugs appear annually and new classes of drugs are invented approximately every ten years still a lot of type 2 diabetic patients remain to be out of the target glycemic levels.

According to most of the guidelines for type 2 diabetes,treatment metformin is the first line therapy for this disease. The choice of second-line antidiabetic drug usually depends on doctors' preference. That is why defining the correct drug for exact patient is still an urgent question. This review provides data on antidiabetic drugspotential for preventing the progression of micro- and macrovascular complications. The question of the potential of early antidiabetic therapy intensification to activate legacy effect is debated. Early and lasting compensation of diabetes with the use of multiple drugs can become a basis for primary prevention of cardiovascular disease in such patients.

KEYWORDS: type 2 diabetes; hypoglycemic drugs; DPP-4 inhibitors; SGLT-2 inhibitors; microvascular complications;macrovascular complications; legacy effect

В настоящее время в арсенале диабетологов имеется большой выбор сахароснижающих препаратов. Консенсус двух крупнейших организаций - Американской и Европейской диабетологических ассоциаций ADA/EASD [1] рекомендует назначение метформина в качестве первой линии терапии. В качестве второй линии при недостижении целевого значения уровня гликированного гемоглобина $\left(\mathrm{HbA}_{1 c}\right)$ могут быть выбраны:

ингибиторы дипептидилпептидазы 4 типа (иДПП-4);

ингибиторы натрийзависимого глюкозного ко-транспортера 2 типа (иНГЛТ-2);

препараты сульфонилмочевины (СМ);

тиазолидиндионы (ТЗД);

агонисты рецепторов глюкагоноподобного пептида 1

типа (арГПП-1);

базальный инсулин.
Актуальным остается вопрос определения целевых групп пациентов для того или иного сахароснижающего препарата.

Метформин является эффективным, безопасным, недорогим препаратом для коррекции гипергликемии, в связи с чем именно этот препарат занимает первую позицию среди всех сахароснижающих препаратов [2]. В качестве второй линии может быть назначен любой препарат, не имеющий ограничения для создания комбинации с имеющимся. Редкие сравнительные исследования комбинаций препаратов не позволяют сделать вывод об оптимальном препарате второй линии.

Одним из исследований, ставящим задачу оценить эффективность комбинаций препаратов, является исследование GRADE (рис. 1). Это исследование планирует охватить 5000 пациентов, получающих метформин, с длительностью 

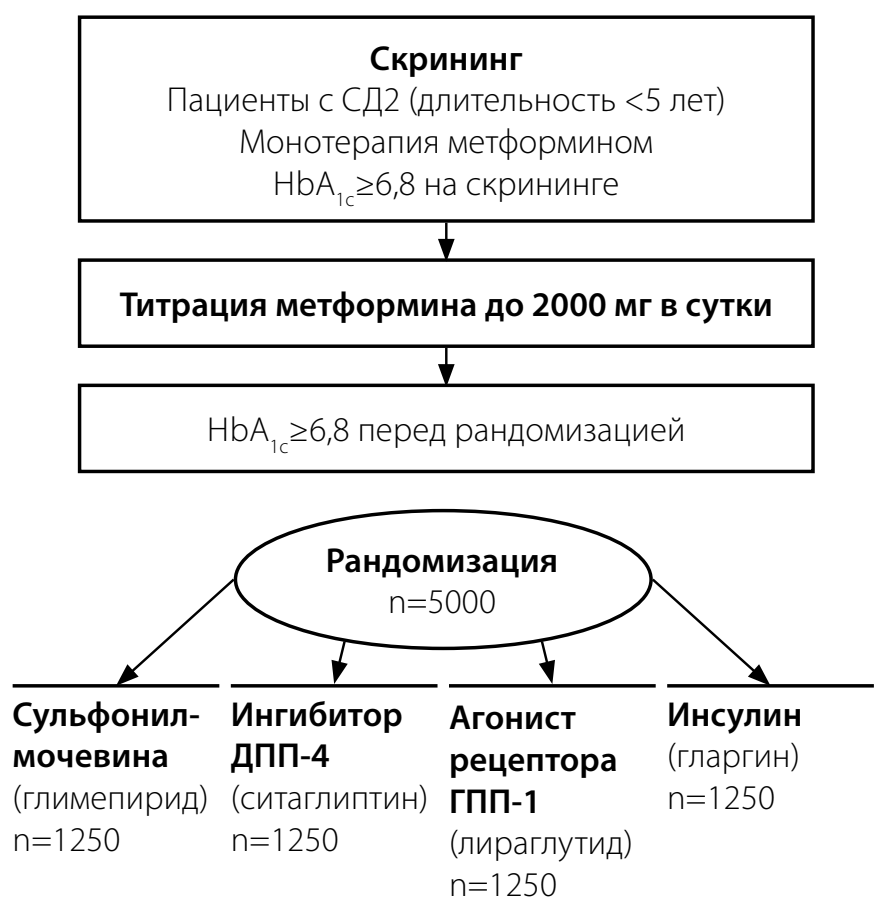

Рис. 1. Дизайн исследования GRADE.

сахарного диабета 2 типа (СД2) менее 5 лет и исходным уровнем $\mathrm{HbA}_{1 с} 6,8-8,5 \%$ [3].

В исследовании будет изучена сахароснижающая эффективность 4 комбинаций метформина: с иДПП-4, препаратами СМ, арГПП-1 и базальным инсулином. Комбинация метформина с иНГЛТ-2 не присутствует в этом исследовании, так как на момент начала исследования данный класс препаратов только входил на рынок. Результаты исследования GRADE позволят оценить эффективность различных комбинаций сахароснижающих препаратов, их переносимость и экономическую составляющую.

Тем не менее, пока не получены результаты сравнительных исследований, отсутствуют четкие критерии выбора препарата второй и третьей линии. С одной стороны, это предоставляет возможность индивидуального подхода к лечению пациентов с СД2, с другой - затрудняет выбор врачом наиболее оптимальной терапевтической стратегии.

Рекомендации ADA/EASD предлагают основывать свой выбор на ряде критериев эффективности и безопасности сахароснижающих препаратов: степени снижения уровня гликированного гемоглобина, риске гипогликемий, влиянии на вес, профиле побочных эффектов и стоимости [4].

Исходя из профиля безопасности и удобства приема препаратов, наиболее часто используемыми комбинациями в настоящий момент являются комбинации метформин+иДПП-4 и метформин+иНГЛТ-2. На возможностях этих двух комбинаций в профилактике осложнений СД2 остановимся подробнее.

\section{ВЛИЯНИЕ ИДДП-4 И ИНГЛТ-2 НА МИКРОСОСУДИСТЫЕ ОСЛОЖНЕНИЯ САХАРНОГО ДИАБЕТА (НЕФРОПАТИЯ)}

Хроническая болезнь почек (ХБП) встречается почти у 40\% пациентов с СД2 [5]. Течение ХБП усугубляется при наличии таких факторов риска, как ожирение и сердечно-сосудистые заболевания. По данным статистики, СД является основной причиной развития терминальной почечной недостаточности [6].
Фармакологическая профилактика и лечение диабетической нефропатии включают воздействие на три основных фактора: гипергликемию, артериальную гипертензию и дислипидемию. В контроле артериальной гипертензии преимущество отдается препаратам, блокирующим ренин-ангиотензин-альдостероновую систему, целевые значения АД у большинства лиц с СД не превышают 140/85 мм рт.ст. [7]. У лиц с 3-й степенью альбуминурии следует добиваться более выраженного снижения систолического АД менее 130/85 мм рт. ст.

Важное значение имеет коррекция дислипидемии на ранних стадиях развития диабетической нефропатии, так как при прогрессировании ХБП защитный эффект от снижения ЛПНП теряется [8]. Тем не менее, комбинированный риск сердечно-сосудистых событий и терминальной почечной недостаточности прогрессивно увеличивается при снижении СКФ и нарастании альбуминурии, поэтому всем пациентам с диабетической нефропатией рекомендован прием липидснижающей терапии.

Снижение гликемии является одним из приоритетных направлений в профилактике развития и прогрессирования поражения почек при СД. Препараты класса иДПП-4 продемонстрировали свою безопасность при любой степени снижения скорости клубочковой фильтрации (СКФ). Все препараты, кроме линаглиптина, требуют коррекции дозы при умеренной-тяжелой ХБП [7].

Помимо безопасности, иДПП-4 обладают свойством снижать степень альбуминурии. В экспериментальных работах линаглиптин в комбинации с блокатором рецептора ангиотензина телмисартаном продемонстрировал снижение выраженности альбуминурии у мышей, лишенных гена синтазы эндотелиального оксида азота (eNOS) [9]. Выбранная модель животных имитирует развитие эндотелиальной дисфункции - одного из важнейших патогенетических путей формирования диабетической нефропатии. В данном исследовании терапия линаглиптин+телмисартан позволяла достичь значительного снижения степени альбуминурии по сравнению с монотерапией телмисартаном. Важно, что протективный эффект линаглиптина наблюдался вне зависимости от выраженности снижения гликемии и уровня артериального давления.

Аналогичное исследование было проведено среди пациентов с СД2, когда этим лицам с персистирующей микроальбуминурией (альбуминурией 2-й степени) совместно с блокатором рецепторов к ангиотензину назначались различные иДПП-4 [10]. В этом исследовании на фоне назначения алоглиптина снижение альбуминурии было наиболее выраженным.

В целом группа инкретиннаправленной терапии демонстрирует нефропротективные свойства: имеются данные об уменьшении ретенции натрия, ингибировании синтеза фиброгенных медиаторов, воспалительных сигнальных путей, процессов свободнорадикального окисления и апоптоза нефроцитов на фоне данных препаратов [11]. Проспективным исследованием по изучению нефропротективной активности иДПП-4 станет многоцентровое плацебо-контролируемое PKИ MARLINA-T2DTM [12]. Пациенты с недостаточным контролем углеводного обмена и ХБП будут рандомизированы в равные группы, одна из которых будет получать в течение 24 нед линаглиптин 5 мг в добавление к существующей терапии, вторая - плацебо. Конечными точками исследования являются уровень гли- 
кированного гемоглобина $\left(\mathrm{Hb}_{1 c}\right)$ и отношение альбумин/ креатинин мочи.

Согласно «Алгоритмам специализированной помощи больным сахарным диабетом», при выраженном снижении СКФ предпочтение отдается иДПП-4, так как эти препараты могут применяться на любой стадии ХБП с учетом коррекции дозы [7].

Нефропротективными свойствами обладают и иНГЛТ-2: эти препараты снижают внутриклубочковое давление и выраженность альбуминурии. По данным исследования EMPA-REG OUTCOME, назначение эмпаглифлозина снижало вероятность развития и прогрессирования нефропатии на 39\% [13]. Применение канаглифлозина по сравнению с плацебо в исследовании CANVAS снижало на 27\% прогрессирование альбуминурии и на 40\% комбинированный показатель - ухудшение СКФ, необходимость в заместительной почечной терапии, смерть по причине ХБП [14]. Однако применение иНГЛТ-2 не разрешено у лиц с СКФ менее 45-60 мл/мин/1,73 м². Более того, в исследовании CANVAS терапия канаглифлозином приводила к увеличению числа малых ампутаций нижних конечностей.

\section{ВЛИЯНИЕ ИДДП-4 И ИНГЛТ-2 НА МИКРОСОСУДИСТЫЕ ОСЛОЖНЕНИЯ САХАРНОГО ДИАБЕТА (РЕТИНОПАТИЯ)}

Несмотря на расширение терапевтических возможностей лечения СД2, диабетическая ретинопатия (ДР) остается распространенным осложнением этого заболевания [15]. Потенциал воздействия иДПП-4 на течение ДР обусловлен наличием у этой группы препаратов большого числа субстратов, помимо ГПП-1. Одним из таких субстратов является stromalcell-derivedfactor-1 alpha (SDF-1a), отвечающий за ангиогенез [16]. Этот хемокин провоцирует ангиогенез, а концентрация SDF-1а увеличивается при пролиферативной ДР. С другой стороны, продукты деградации ГПП-1 способствуют уменьшению продукции митохондриями активных форм кислорода. Таким образом, иДПП-4 могут двояко влиять на неоваскуляризацию сетчатки: с одной стороны, увеличивая концентрацию SDF-1a, с другой - уменьшая продукцию активных форм кислорода.

В экспериментальном исследовании на мышах линаглиптин оказывал протективный эффект на сетчатку глаза [17]. Частота развития ДР, по данным немецкого регистра, была существенно ниже у пациентов, принимающих вилдаглиптин по сравнению с препаратами СМ (ОШ=0,55, $\mathrm{P}=0,0004)[18]$.

В исследовании EMPA-REG OUTCOME вторичной конечной точкой была частота микрососудистых осложнений: необходимость в проведении ретинальной фотокоагуляции, витреальные геморрагии, слепота, появление или прогрессирование нефропатии. Назначение эмпаглифлозина позволило снизить риск возникновения микрососудистых осложнений, однако преимущественно за счет замедления прогрессирования нефропатии, а не ретинопатии [19].

\section{ВЛИЯНИЕ САХАРОСНИЖАЮЩИХ ПРЕПАРАТОВ НА СЕРДЕЧНО-СОСУДИСТЫЕ ИСХОДЫ}

Главной причиной смертности пациентов с СД являются сердечно-сосудистые исходы. Начало XXI века ознаменовано появлением данных о влиянии сахароснижающих препаратов на течение сердечно-сосудистых заболеваний (СС3). С этого времени наличие ССЗ стало одним из критериев выбора сахароснижающего препарата.

Хорошо доказана связь снижения уровня гликированного гемоглобина и уменьшения числа микрососудистых осложнений СД [20]. Микро- и макрососудистые осложнения находятся в тесной взаимосвязи: пациенты с пролиферативной ДР имеют в 25 раз повышенный риск ампутации нижних конечностей и в 5 раз больший риск развития ишемической болезни сердца [21].

Чем выше уровень $\mathrm{HbA}_{1 с}$ у пациентов с СД2, тем выше риски макрососудистых осложнений: увеличение $\mathrm{HbA}_{1 с}$ на 1\% приводит к росту числа СС3 на 18\% [22].

Тем не менее, снижение уровня гликированного гемоглобина не позволяет достичь таких же преимуществ в уменьшении риска макрососудистых осложнений, как это наблюдается с микрососудистыми осложнениями: исследования по снижению сердечно-сосудистого риска путем более интенсивного контроля гликемии демонстрируют крайне противоречивые результаты [23].

Для объяснения такого парадоксального отсутствия снижения макрососудистых осложнений по сравнению с микрососудистыми при равной степени снижения $\mathrm{HbA}_{1 с}$ предлагается множество объяснений: потенцирование гипогликемии рядом сахароснижающих препаратов, увеличение массы тела, иные побочные эффекты сахароснижающей терапии, неверно выбранная цель снижения $\mathrm{HbA}_{1 c^{\prime}}$ недостаточная длительность исследования и др. Одна из реалистичных причин такого парадокса может крыться в самой мультифакториальной природе макрососудистых осложнений, риск которых при СД2 зависит не только и не столько от степени гипергликемии, но, как показали результаты исследования STENO-2, и от выраженности артериальной гипертензии и дислипидемии [24].

В 2008 г. после отзыва росиглитазона с рынка Управление по санитарному надзору за качеством пищевых продуктов и медикаментов (FDA) издало нормативный акт, согласно которому, все новые препараты для лечения СД2 должны иметь исследования по сердечно-сосудистой безопасности [25].

К настоящему моменту закончено уже восемь исследований по сердечно-сосудистой безопасности сахароснижающих препаратов: три исследования иДПП-4 (SAVORTIMI 53, EXAMINE, TECOS), три исследования арГПП-1 (ELIXA, LEADER, SUSTAIN-6) и два исследования инГЛТ-2 (EMPA-REG OUTCOME, CANVAS).

Первыми завершились исследования иДПП-4. Все три достигли критериев безопасности терапии по комбинированной конечной точке (сердечно-сосудистая смертность, нефатальный ИМ, нефатальный инсульт). В исследовании SAVOR-TIMI 53 наблюдалось увеличение числа госпитализаций по поводу сердечной недостаточности (CH) у пациентов, принимающих иДПП-4 саксаглиптин по сравнению с плацебо, несмотря на отсутствие увеличения сердечно-сосудистой смертности [26]. Данные результаты насторожили общественность в отношении других иДПП-4, но алоглиптин в исследовании EXAMINE не продемонстрировал статистически значимого увеличения риска госпитализаций по поводу СН, несмотря на то, что этот препарат исследовался в группе пациентов наиболее высокого риска (перенесших острый коронарный синдром от 15 до 90 дней до включения в исследование) [27]. Еще один 
иДПП-4 - ситаглиптин в исследовании TECOS также не увеличивал риск СН среди пациентов с имеющимися кардиоваскулярными заболеваниями [28]. Поэтому в целом класс иДПП-4 считается безопасным для лиц с СС3 в анамнезе.

Первое исследование сердечно-сосудистой безопасности арГПП-1 ликсисенатида продемонстрировало нейтральный эффект данного препарата в отношении снижения сердечно-сосудистого риска по сравнению с плацебо [29]. А последовавшие за этим два других исследования арГПП-1 показали превосходство представителей данного класса: назначение лираглутида позволило снизить комбинированную конечную точку (сердечно-сосудистая смертность/ИМ/инсульт) на 13\%, преимущественно за счет снижения сердечно-сосудистой смертности, а назначение семаглутида - на 26\%, преимущественно за счет снижения числа инсультов [30, 31].

Наконец, исследование EMPA-REG OUTCOME стало первым по оценке сердечно-сосудистой безопасности иНГЛТ-2 эмпаглифлозина у пациентов с имеющимися СС3 и продемонстрировало снижение комбинированной конечной точки на 14\%, при этом снижение сердечно-сосудистой смертности составило 38\%, а риска госпитализаций по поводу декомпенсации СН - 35\% [32].

В исследовании CANVAS [14] назначение еще одного представителя класса иНГЛТ-2 привело к снижению комбинированной конечной точки (СС-смерть, нефатальный ИМ, нефатальный инсульт) на 14\%.

Но все предложенные исследования - исследования вторичной профилактики сердечно-сосудистых исходов. Поэтому корректно экстраполировать результаты данных исследований на выборку пациентов с уже имеющимися CC3 атеросклеротического генеза или пациентов высокого сердечно-сосудистого риска. Доказательств преимущества иНГЛТ-2 или арГПП-1 у пациентов, не соответствующих критериям включения в исследования EMPA-REG OUTCOME, LEADER, SUSTAIN-6, на настоящий момент нет.

\section{ПЕРВИЧНАЯ ПРОФИЛАКТИКА СЕРДЕЧНО-СОСУДИСТЫХ ИСХОДОВ У ПАЦИЕНТОВ С СД2 КРОЕТСЯ В АКТИВАЦИИ МЕТАБОЛИЧЕСКОЙ ПАМЯТИ?}

Под термином «метаболическая память» понимают сохранение эффекта предшествующего гликемического контроля (хорошего или неудовлетворительного) на развитие и прогрессирование сосудистых осложнений при СД [33]. У больных СД2 феномен «метаболической памяти» был впервые продемонстрирован в исследовании UKPDS (United Kingdom Prospective Diabetes Study), длившемся на протяжении 20 лет (1977-1997) [34] и затем продолженном в 10-летнем наблюдении. В это исследование исходно были включены больные СД2 с впервые установленным диагнозом без сердечно-сосудистых осложнений. Пациенты были разделены на две группы: получающие интенсивную медикаментозную сахароснижающую терапию (метформином, препаратами СМ или инсулина) или получающие традиционную диетотерапию без добавления медикаментозных средств, что на тот момент являлось стандартом лечения. В ходе исследования в среднем за 10 лет наблюдения в группе интенсивной терапии показатель $\mathrm{HbA}_{1 c}$ оказался на 0,9\% ниже, чем в группе сравнения (7,0\% против 7,9\% соответственно), что сопровождалось значимым снижением риска микрососудистых осложнений на
25\%, но отсутствием достоверного снижения риска сердечно-сосудистых исходов, инфаркта миокарда и смертности от всех причин. Далее пациенты продолжили участие в проекте в рамках наблюдательного исследования, в результате чего в течение последующих 10 лет показатели контроля гликемии в обеих группах сравнялись, в конце исследования уровень $\mathrm{HbA}_{1 с}$ в обеих группах составил около 8\%. Однако, невзирая на ухудшение гликемического контроля, риски микрососудистых осложнений в группе ранее получавших интенсивную терапию по-прежнему оставались достоверно ниже, чем в группе стандартного лечения. Более того, через дополнительные 10 лет наблюдения в «интенсивной»группе снижение рисков инфаркта миокарда и летальности от всех причин впервые достигло статистически значимой разницы [35].

Эти исследования доказали, что ранний эффективный старт сахароснижающей терапии позволяет достичь не только профилактики микрососудистых, но и макрососудистых осложнений. Приняв такую концепцию во внимание, объяснимы и «провальные» результаты исследования ACCORD. В интенсивной ветви исследования ACCORD не только не отмечалось снижения риска микро- и макрососудистых осложнений, а наоборот, наблюдалось повышение смертности от сердечно-сосудистых причин, что повлекло за собой преждевременную остановку исследования и тщательный анализ данных с целью выявить возможные причины таких результатов исследования [36]. В то же время, если проанализировать исходные характеристики включенных пациентов, то станет очевидно принципиальное отличие этого исследования от UKPDS: в исследование ACCORD были включены пациенты с длительным анамнезом плохо контролируемого СД. Таким образом, «метаболическая память» может не только улучшить прогноз пациента в случае длительной компенсации в дебюте заболевания, но и сыграть злую шутку тогда, когда на старте болезни такой компенсации достигнуто не было.

Таким образом, успешная коррекция метаболических нарушений у пациентов с СД2 может являться ключом к активации «метаболической памяти» и служить основой для реализации первичной профилактики сердечно-сосудистых исходов у таких больных.

Для доказательства возможности первичной профилактики СС3 у пациентов с СД2 потребуются длительные исследования, включающие пациентов без СС3 в анамнезе. Для изучения влияния сахароснижающей терапии на конечные точки (смертность, инфаркт миокарда, острое нарушение мозгового кровообращения (ОНМК)) у лиц без исходных СС3 должны пройти десятилетия, поэтому среди данной когорты больных чаще изучаются факторы риска или суррогатные точки. В частности получены результаты исследования SPEAD-A, изучавшего влияние сахароснижающей терапии на толщину комплекса интима-медиа [37]. В данном исследовании применение иДПП- алоглиптина позволяло более значимо предотвращать прогрессию утолщения комплекса интима-медиа по сравнению с другими сахароснижающими препаратами у лиц без ССЗ в анамнезе.

Риск развития гипогликемии в течение последних нескольких лет вышел на передний план как основной ограничивающий фактор в интенсификации терапии в связи с повышением риска смерти, наблюдаемым в исследовании ACCORD. Результаты этого исследования заставили пересмотреть цели сахароснижающей терапии, в связи с чем 


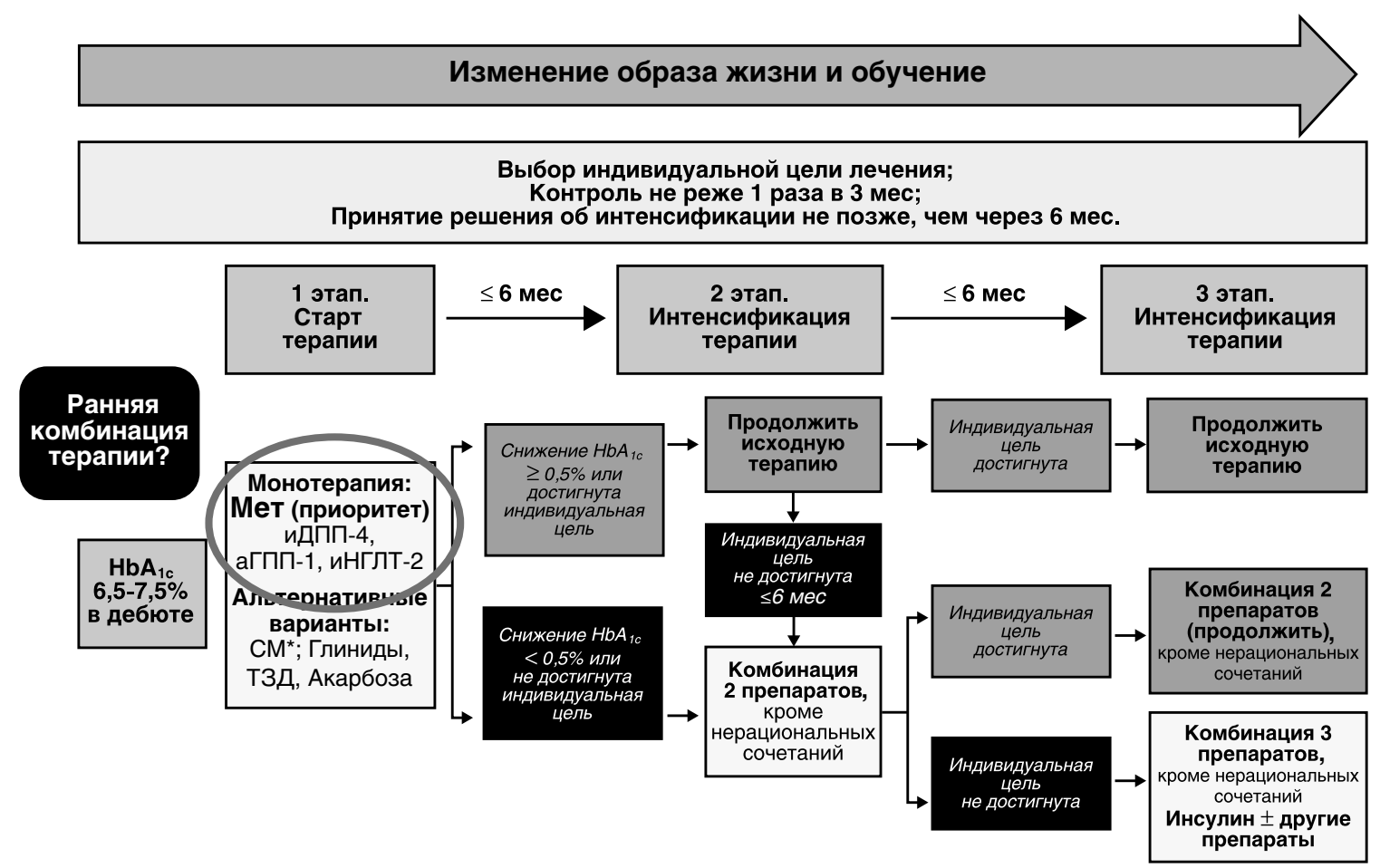

Рис. 2. Возможное место ранней комбинированной терапии в алгоритмах помощи больным СД2 (адаптировано из [7]).

пациентам с макрососудистыми осложнениями и высоким риском гипогликемий рекомендуются крайне гибкие цели компенсации углеводного обмена [7].

B то же время с момента проведения исследования ACCORD на рынке появились новые классы сахароснижающих препаратов, обладающих низким риском гипогликемий: иДПП-4, арГПП-1 и иНЛГТ-2. Возникает закономерный вопрос, были бы негативными результаты исследования ACCORD, если бы используемые препараты в группе интенсивной терапии обладали невысоким риском гипогликемий? И если нет, то можно ли вновь ужесточить требования к компенсации углеводного обмена, учитывая благоприятный профиль действия новых сахароснижающих препаратов?

Ответы на эти вопросы пока остаются открытыми, но возможности управления СД с появлением новых сахароснижающих препаратов, безусловно, расширились.

\section{РАННЯЯ КОМБИНАЦИЯ САХАРОСНИЖАЮЩИХ ПРЕПАРАТОВ - БЕЗОПАСНЫЙ ПУТЬ К РЕАЛИЗАЦИИ МЕТАБОЛИЧЕСКОЙ ПАМЯТИ?}

Российские «Алгоритмы специализированной медицинской помощи больным сахарным диабетом» (2017 г.) предлагают в качестве первой линии терапии у пациентов с исходным уровнем $\mathrm{HbA}_{1 с} 6,5 \%-7,5 \%$ альтернативу из монотерапии метформином, иДПП-4, арГПП-1 или иНГЛТ-2 (при непереносимости и противопоказаниях к назначению этих групп могут быть назначены препараты СМ, глиниды, ТЗД, акарбоза) [7].

Как уже говорилось, для активации метаболической памяти необходимо с дебюта заболевания на протяжении длительного времени поддерживать целевой гликемический контроль: длительная компенсация с начала заболевания позволяет снизить риск микрососудистых осложнений, а при длительной компенсации - и макрососудистых осложнений.
Десятилетняя оценка динамики $\mathrm{HbA}_{1 с}$ у 28000 пациентов показала, что наилучшей профилактики микро- и макрососудистых осложнений удается достичь тогда, когда уровень НbА в в течение времени остается стабильным [38]. При этом одинаково неблагоприятны варианты чередования высокого и низкого $\mathrm{HbA}_{1 c^{\prime}}$ даже тогда, когда в дебюте заболевания $\mathrm{HbA}_{1 с}$ высок, а затем быстро приходит к целевым значениям. Современная тактика управления СД2 основывается на эскалации терапии от монотерапии до комбинированной терапии пероральными сахароснижающими препаратами и/или инсулином. В таких условиях интенсификация терапии происходит тогда, когда предыдущий вариант терапии не позволяет достичь цели. Поэтому возможности длительного поддержания стабильных значений $\mathrm{HbA}_{1 с}$ крайне ограничены.

Способом преодоления «виражей» $\mathrm{HbA}_{1 с}$ могла бы стать ранняя комбинированная терапия. Мета-анализ 62 исследований, включавших 25 сахароснижающих молекул, ставил перед собой задачу оценить степень снижения $\mathrm{HbA}_{1 с}$ на фоне добавления различных препаратов к исходной терапии метформином. [39]. Ингибиторы ДПП-4 и иНГЛТ-2 продемонстрировали сходную эффективность по снижению $\mathrm{HbA}_{1 с}$ в пределах от -0,5 до -0,68 (среди иДПП-4) и от -0,43 до -0,67 (среди иНГЛТ-2). Наиболее эффективной тройной комбинацией были алоглиптин/пиоглитазон/метформин, позволявшей достичь снижения $\mathrm{HbA}_{1 с}$ на -1,24\%, и эмпаглифлозин/линаглиптин/метформин, продемонстрировавшей снижение $\mathrm{HbA}_{1 с}$ на $-1,13 \%$.

Рациональной представляется комбинация метформина с иДПП-4 и иНГЛТ-2 (рис. 2). В монотерапии каждый из представителей этих групп обладает сопоставимой сахароснижающей способностью: от -0,8 до -0,9\% для иНГЛТ, от -2 до -0,5--1,0\% для иДПП-4, от -1 до -2\% для метформина [7].

Тройная комбинация иДПП-4/иНГЛТ-2 в добавление к терапии метформином позволяет снизить $\mathrm{HbA}_{1 с}$ на 1,1-1,5\% [40]. Препараты действуют на разные звенья 
патогенеза СД2 и дополняют друг друга по механизму действия, а также обладают благоприятным профилем переносимости: не увеличивают вес, не вызывают гипогликемий, уравновешивают концентрацию глюкагона [41]. Помимо этого, все три группы препаратов способны снижать риск микрососудистых осложнений СД2. Таким образом, двойная комбинация метформин + иДПП-4/иНГЛТ-2 или тройная комбинация этих препаратов обладает всеми возможностями для активации метаболической памяти и улучшения прогноза пациентов с СД2. Требуются длительные исследования для определения безопасности и эффективности ранней комбинированной терапии у пациентов с СД2.

\section{ДОПОЛНИТЕЛЬНАЯ ИНФОРМАЦИЯ}

Финансирование работы. Поисково-аналитическая работа проведена в рамках выполнения государственного задания «Взаимодействие генетических, метаболических и воспалительных факторов развития и прогрессирования сосудистых осложнений сахарного диабета, в том числе после хирургически индуцированной ремиссии диабета» (НИOКТР AАAА-А17-117012610110-9).

Конфликт интересов. Е.А. Шестакова - лектор, участник экспертных советов компаний AstraZeneka, Boeringer Ingelheim, EliLilly, MSD, Novartis, NovoNordisk, Takeda.

\section{СПИСОК ЛИТЕРАТУРЫ| REFERENCES}

1. American Diabetes Association. Pharmacologic Approaches to Glycemic Treatment. Diabetes Care. 2017;40(Suppl 1):64-74. doi: 10.2337/dc17-S011

2. American Diabetes Association. Standards of medical care in diabetes-2016: summary of revisions. Diabetes Care. 2016;39(Suppl 1):4-5. doi: 10.2337/dc16-S003

3. Nathan DM, Buse JB, Kahn SE, et al. Rationale and design of the glycemia reduction approaches in diabetes: a comparative effectiveness study (GRADE). Diabetes Care. 2013;36(8):2254-2261. doi:10.2337/dc13-0356

4. Inzucchi SE, Bergenstal RM, Buse JB, et al. Management of hyperglycemia in type 2 diabetes, 2015: a patient-centered approach: update to a position statement of the American Diabetes Association and the European Association for the Study of Diabetes. Diabetes Care. 2015;38(1):140-149. doi: 10.2337/dc14-2441

5. Schernthaner $G$, Mogensen $C E$, Schernthaner $G H$. The effects of GLP-1 analogues, DPP-4 inhibitors and SGLT2 inhibitors on the renal system. Diab Vasc Dis Res. 2014;11 (5):306-323. doi: 10.1177/1479164114542802

6. U.S. Renal Data System. USRDS 2012 annual data report: atlas of chronic kidney disease and end-stage renal disease in the United States. Bethesda: National Institutes of Health, National Institute of Diabetes and Digestive and Kidney Diseases; 2012. Available from: https://www.usrds.org/atlas12.aspx.

7. Дедов И.И., Шестакова М.В., Майоров А.Ю., и др. Алгоритмы специализированной медицинской помощи больным сахарным диабетом / Под редакцией И.И. Дедова, М.В. Шестаковой, А.Ю. Майорова. - 8-й выпуск // Сахарный диабет. - 2017. - Т. 20. №1S. - C. 1-121. [Dedov II, Shestakova MV, Mayorov AY, et al. Standards of specialized diabetes care. Edited by Dedov II, Shestakova MV, Mayorov AY. 8th edition. Diabetes mellitus. 2017;20(1S):1-121. (In Russ.)] doi: 10.14341/DM8146

8. Athyros VG, Mitsiou EK, Tziomalos K, et al. Impact of managing atherogenic dyslipidemia on cardiovascular outcome across different stages of diabetic nephropathy. Expert Opin Pharmacother. 2010;11(5): 723-730. doi: 10.1517/14656560903575654

9. Alter ML, Ott IM, von Websky K, et al. DPP-4 inhibition on top of angiotensin receptor blockade offers a new therapeutic approach for diabetic nephropathy. Kidney Blood Press Res. 2012;36(1):119-130. doi: 10.1159/000341487

10. Fujita $\mathrm{H}$, Tania $\mathrm{H}$, Murayama $\mathrm{H}$ et al. DPP-4 inhibition with alogliptin on top of angiotensin II type 1 receptor blockade ameliorates albuminuria via up-regulation of SDF-1 a in type 2 diabetic patients with incipient nephropathy. Endocrine Journal. 2014;61 (2):159-166. doi: 10.1507/endocrj.EJ13-0305

11. Корбут А.И., Климонтов В.В. Терапия, основанная на инкретинах: почечные эффекты // Сахарный диабет. - 2016. - Т. 19. №1. - C. 53-63. [Korbut Al, Klimontov WV. Incretin-based therapy: renal effects. Diabetes mellitus. 2016;19(1):53-63. (In Russ.)] doi: $10.14341 / D M 7727$

12. Groop PH, Cooper ME, Perkovic V, et al. Dipeptidyl peptidase-4 inhibition with linagliptin and effects on hyperglycaemia and albuminuria in patients with type 2 diabetes and renal dysfunction: Rationale and design of the MARLINA-T2DTM trial. Diab Vasc Dis Res. 2015;12(6):455-462. doi: 10.1177/1479164115579002
13. Wanner C, Inzucchi SE, Lachin JM, et al. Empagliflozin and Progression of Kidney Disease in Type 2 Diabetes. N Engl J Med. 2016;375(4):323-334. doi: 10.1056/NEJMoa1515920

14. Neal B, Perkovic V, Mahaffey KW, et al. Canagliflozin and Cardiovascular and Renal Events in Type 2 Diabetes. N Engl J Med. 2017;377:644-657. doi: 10.1056/NEJMoa1611925

15. Дедов И.И., Шестакова М.В., Викулова О.К. Эпидемиология сахарного диабета в Российской Федерации: клинико-статистический анализ по данным Федерального регистра сахарного диабета // Сахарный диабет. - 2017. - Т. 20. - №1 . - C. 13-41 [Dedov II, Shestakova MV, Vikulova OK. Epidemiology of diabetes mellitus in Russian Federation: clinical and statistical report according to the federal diabetes registry. Diabetes mellitus. 2017;20(1):13-41. (In Russ.)] doi: $10.14341 / D M 8664$

16. Butler JM, Guthrie SM, Koc M, et al. SDF-1 is both necessary and sufficient to promote proliferative retinopathy. $J$ Clin Invest. 2005;115(1):86-93. doi: 10.1172/JCI22869

17. Dietrich N, Kolibabka M, Busch S, et al. The DPP4 Inhibitor Linagliptin Protects from Experimental Diabetic Retinopathy. PLoS One. 2016;11(12):e0167853. doi: 10.1371/journal.pone.0167853

18. Kolaczynski WM, Hankins M, Ong SH, et al. Microvascular Outcomes in Patients with Type 2 Diabetes Treated with Vildagliptin vs. Sulfonylurea: A Retrospective Study Using German Electronic Medical Records. Diabetes Ther. 2016;7(3):483-496 doi: 10.1007/s13300-016-0177-8

19. Wanner C, Lee C, Woerle HJ, et al. Empagliflozin and microvascular outcomes in EMPA-REG OUTCOME. Diabetologia. 2016;59(Suppl 1):483-484. doi: 10.1007/s00125-016-4046-9

20. Stratton IM, Adler Al, Neil HA, et al. Association of glycaemia with macrovascular and microvascular complications of type 2 diabetes (UKPDS 35): prospective observational study. BMJ. 2000;321(7258):405-412. doi: 10.1136/bmj.321.7258.405

21. Juutilainen $A$, Lehto $S$, Ronnemaa $T$, et al. Retinopathy predicts cardiovascular mortality in type 2 diabetic men and women. Diabetes Care. 2007;30(2):292-299. doi: 10.2337/dc06-1747

22. Emerging Risk Factors Collaboration; Di Angelantonio E, Gao P, et al. Glycated hemoglobin measurement and prediction of cardiovascular disease. JAMA. 2014;311(12):1225-33. doi: 10.1001/jama.2014.1873

23. Boussageon R, Bejan-Angoulvant T, Saadatian-Elahi M, et al. Effect of intensive glucose lowering treatment on all cause mortality, cardiovascular death, and microvascular events in type 2 diabetes: meta-analysis of randomised controlled trials. BMJ. 2011;343:d4169. doi: 10.1136/bmj.d4169

24. Gaede $P$, Vedel $P$, Larsen $N$, et al. Multifactorial intervention and cardiovascular disease in patients with type 2 diabetes. N Eng J Med. 2003;348(5):383-393. doi: 10.1056/NEJMoa021778

25. Guidance for Industry. Diabetes mellitus_evaluating cardiovascular risk in new antidiabetic therapies to treat type 2 diabetes. 2008. Silver Spring: U.S. Department of Health and Human Services, Food and Drug Administration, Center for Drug Evaluation and Research (CDER); 2008. Available from: https://www.fda.gov/downloads/ Drugs/../Guidances/ucm071627.pdf.

26. Scirica BM, Bhatt DL, Braunwald E, et al. Saxagliptin and cardiovascular outcomes in patients with type 2 diabetes mellitus. N Eng J Med. 2013;369(14):1317-1326. doi: 10.1056/NEJMoa1307684 
27. White WB, Cannon CP, Heller SR, et al. Alogliptin after acute coronary syndrome in patients with type 2 diabetes. N Eng J Med. 2013;369(14):1327-1335. doi: 10.1056/NEJMoa1305889

28. Green JB, Bethel MA, Armstrong PW, et al. Effect of sitagliptin on cardiovascular outcomes in type 2 diabetes. N Eng J Med. 2015;373(3):232-242. doi: 10.1056/NEJMoa1501352

29. Pfeffer MA, Claggett B, Diaz R, et al. Lixisenatide in patients with type 2 diabetes and acute coronary syndrome. N Eng J Med. 2015;373(23):2247-2257. doi: 10.1056/NEJMoa1509225

30. Marso SP, Daniels GH, Brown-Frandsen K, et al. Liraglutide and cardiovascular outcomes in type 2 diabetes. N Eng J Med. 2016;375(4):311-322. doi: 10.1056/NEJMoa1603827

31. Marso SP, Bain SC, Consoli A, et al. Semaglutide and cardiovascular outcomes in patients with type 2 diabetes. N Eng/ J Med. 2016;375(19):1834-1844. doi: 10.1056/NEJMoa1607141

32. Zinman B, Wanner C, Lachin JM, et al. Empagliflozin, cardiovascular outcomes, and mortality in type 2 diabetes. N Eng J Med. 2015;373(22):2117-2128. doi: 10.1056/NEJMoa1504720

33. Дедов И.И., Шестакова М.В. Феномен «метаболической памяти» в прогнозировании риска развития сосудистых осложнений при сахарном диабете // Терапевтический архив. - 2015. - Т. 87. - №10. C. 4-10. [Dedov II, Shestakova MV. The metabolic memory phenomenon in predicting a risk for vascular complications in diabetes mellitus. Ter Arkh. 2015:87(10):4-10. doi: 10.17116/terarkh201587104-10

34. Stratton IM, Adler Al, Neil HA, et al. Association of glycaemia with macrovascular and microvascular complications of type 2 diabetes (UKPDS 35): prospective observational study. BMJ. 2000;321(7258):405-412. doi: 10.1136/bmj.321.7258.405
35. Holman RR, Paul SK, Bethel MA, et al. 10-year follow-up of intensive glucose control in type 2 diabetes. N Eng/ J Med. 2008; 359:1577-1589. doi: 10.1056/NEJMoa0806470

36. Action to Control Cardiovascular Risk in Diabetes Study Group; Gerstein HC, Miller ME, Byington RP, et al. Effects of Intensive Glucose Lowering in Type 2 Diabetes. N Engl J Med. 2008;358(24):2545-2559. doi: 10.1056/NEJMoa0802743

37. Mita T, Katakami N, Yoshii H, et al. Alogliptin, a Dipeptidyl Peptidase 4 Inhibitor, Prevents the Progression of Carotid Atherosclerosis in Patients With Type 2 Diabetes: The Study of Preventive Effects of Alogliptin on Diabetic Atherosclerosis (SPEAD-A). Diabetes Care. 2016;39(1):139-148. doi: 10.2337/dc15-0781

38. Laiteerapong N, Karter AJ, Moffet HH, et al. Ten-year hemoglobin A1c trajectories and outcomes in type 2 diabetes mellitus: The Diabetes \& Aging Study. J Diabetes Complications. 2017;31(1):94-100 doi: 10.1016/j.jdiacomp.2016.07.023

39. Mearns ES, Sobieraj DM, White CM, et al. Comparative efficacy and safety of antidiabetic drug regimens added to metformin monotherapy in patients with type 2 diabetes: a network meta-analysis. PLoS One. 2015;10(4):e0125879. doi: 10.1371/journal.pone.0125879

40. Lingvay I. Sodium glucose cotransporter 2 and dipeptidyl peptidase-4 inhibition: promise of a dynamic duo. Endocr Pract. 2017;23(7): 831-840. doi: 10.4158/EP161725.RA

41. Scheen AJ. DPP-4 inhibitor plus SGLT-2 inhibitor as combination therapy for type 2 diabetes: from rationale to clinical aspects. Expert Opin Drug Metab Toxicol. 2016;12(12):1407-1417. doi: 10.1080/17425255.2016.1215427

\section{ИНФОРМАЦИЯ ОБ АВТОРАХ [AUTHORS INFO]}

Шестакова Екатерина Алексеевна, К.М.H. [Ekaterina A. Shestakova, MD, PhD]; адpec: 117036, Mockва, ул. Дм. Ульянова, Д. 11 [address: 11 Dm. Ulyanova str., Moscow, 117036 Russian Federation]; ORCID: http://orcid.org/0000-0001-6612-6851; eLibrary SPIN: 1124-7600; e-mail: katiashestakova@mail.ru.

\section{ЦИТИРОВАТЬ:}

Шестакова Е.А. Выбор второй линии терапии у пациентов с сахарным диабетом 2 типа: активация метаболической памяти // Сахарный диабет. 2017. — T. 20. — №5. - C. 356-362. doi: 10.14341/DM8793

\section{TO CITE THIS ARTICLE:}

Shestakova EA. Second line therapy in type 2 diabetes: legacy effect activation. Diabetes mellitus. 2017;20(5):356-362. doi: 10.14341/DM8793 